\title{
The value of diffusion weighted imaging-alberta stroke program early CT score in predicting stroke-associated pneumonia in patients with acute cerebral infarction: a retrospective study
}

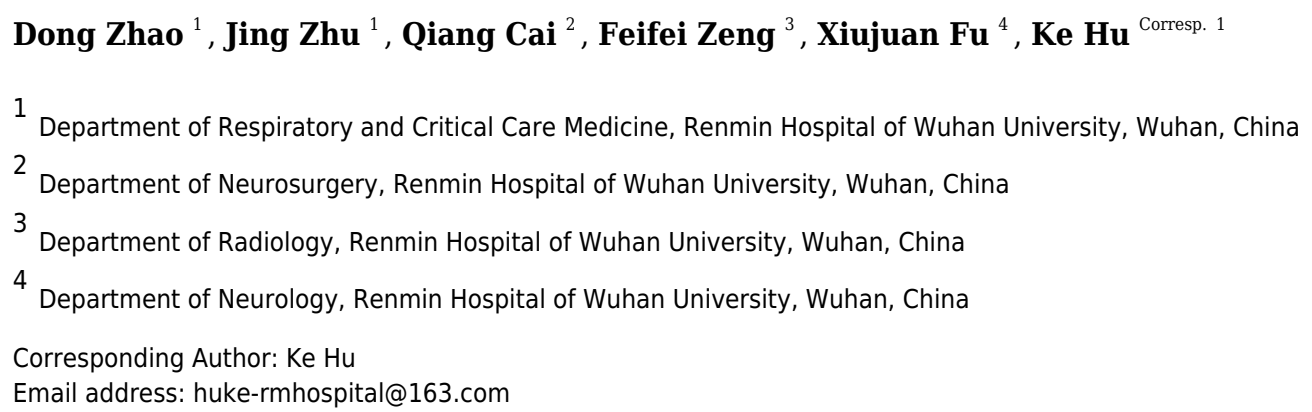

Background. In this study, we aimed to investigate the value of Diffusion-Weighted Imaging-Alberta Stroke Program Early CT Score (DWI-ASPECTS) in predicting strokeassociated pneumonia (SAP) in patients with acute ischemic stroke. Methods. A total of 291 patients who suffered acute cerebral infarction for the first time were included in this retrospective study. DWI-ASPECTS was assessed and clinical data were collected in order to find the risk factors of SAP, and a logistic regression model was used to investigate the effect of predicting SAP. Furthermore, correlation analysis was used to explore the relationship between DWI-ASPECTS and the immume status of the body. Results. Among the 291 patients, 74 (25.4\%) subjects were diagnosed with SAP. Compared with non-SAP, the patients with SAP were older and had a higher rate of atrial fibrillation (AF), National Institutes of Health Stroke Scale (NIHSS) scores. The SAP group also had a significantly lower DWI-ASPECTS than did the non-SAP group $(P<0.01)$. In the multivariable logistic regression analysis, the DWI-ASPECTS (adjusted odds ratio $[\mathrm{aOR}]=1.438 ; 95 \% \mathrm{Cl}$ :

1.158-1.787; $P<0.01)$ remained significant after adjusting for confounders. What's more, the predictive ability of DWI-ASPECTS (AUC $=0.743>0.7,95 \% \mathrm{Cl} 0.678-0.800$ ) had acceptable discriminatory abilities. By the correlation analysis, DWI-ASPECTS was found to be negatively correlated with the count of white blood cell, neutrophils, monocytes, neutrophil-to-monocyte ratio and neutrophil-to-lymphocyte ratio, and positively correlated with the count of lymphocytes. Conclusions. DWI-ASPECTS grades could predict strokeassociated pneumonia for patients with acute ischemic stroke, and combining grade with age, AF, or NIHSS could predict SAP events more accurately. 
1 The value of diffusion weighted imaging-alberta stroke program early CT score in

2 predicting stroke-associated pneumonia in patients with acute cerebral infarction:a

3 retrospective study

4

5

6

7

Dong Zhao ${ }^{1}$, Jing Zhu' ${ }^{1}$, Qiang $\mathrm{Cai}^{2}$, Feifei Zeng ${ }^{3}$, Xiujuan Fu ${ }^{4}$, Ke Hu Corresp. 1

8

9

${ }^{1}$ Department of Respiratory and Critical Care Medicine, Renmin Hospital of Wuhan University, 10 Zhangzhidong Road No. 99, Wuhan, China.

112 Department of Neurosurgery, Renmin Hospital of Wuhan University, Zhangzhidong Road No.

12 99, Wuhan, China.

$13{ }^{3}$ Department of Radiology, Renmin Hospital of Wuhan University, Zhangzhidong Road No. 99, 14 Wuhan, China.

$15{ }^{4}$ Department of Neurology, Renmin Hospital of Wuhan University, Zhangzhidong Road No. 99, 16 Wuhan, China.

17

18 Corresponding Author: $\mathrm{Ke} \mathrm{Hu}$

19 Renmin Hospital of Wuhan University, Zhangzhidong Road No. 99, Wuhan 430060, China.

20 Email address: huke-rmhospital@163.com

21

22

23

24

25

26

27

28

29

30

31

32

33

34

35

36 
38

39

40

41

42

43

44

45

46

47

48

49

50

51

52

53

54

55

56

57

58

59

60

61

62

63

64

65

66

67

68

69

\section{Abstract}

Background. In this study, we aimed to investigate the value of Diffusion-Weighted ImagingAlberta Stroke Program Early CT Score (DWI-ASPECTS) in predicting stroke-associated pneumonia (SAP) in patients with acute ischemic stroke.

Methods. A total of 291 patients who suffered acute cerebral infarction for the first time were included in this retrospective study. DWI-ASPECTS was assessed and clinical data were collected in order to find the risk factors of SAP, and a logistic regression model was used to investigate the effect of predicting SAP. Furthermore, correlation analysis was used to explore the relationship between DWI-ASPECTS and the immune status of the body.

Results. Among the 291 patients, 74 (25.4\%) subjects were diagnosed with SAP. Compared with non-SAP, the patients with SAP were older and had a higher rate of atrial fibrillation (AF), National Institutes of Health Stroke Scale (NIHSS) scores. The SAP group also had a significantly lower DWI-ASPECTS than did the non-SAP group $(\mathrm{P}<0.01)$. In the multivariable logistic regression analysis, the DWI-ASPECTS (adjusted odds ratio $[\mathrm{aOR}]=1.438$; 95\% CI: 1.158-1.787; $\mathrm{P}<0.01)$ remained significant after adjusting for confounders. What's more, the predictive ability of DWI-ASPECTS (AUC $=0.743>0.7,95 \%$ CI $0.678-0.800)$ had acceptable discriminatory abilities. By the correlation analysis, DWI-ASPECTS was found to be negatively correlated with the count of white blood cell, neutrophils, monocytes, neutrophil-to-monocyte ratio and neutrophil-to-lymphocyte ratio, and positively correlated with the count of lymphocytes 0

Conclusions. DWI-ASPECTS grades could predict stroke-associated pneumonia for patients with acute ischemic stroke, and combining grade with age, AF, or NIHSS could predict SAP events more accurately.

Keywords: Ischemic Stroke, Stroke-associated Pneumonia, Diffusion-Weighted ImagingAlberta Stroke Program Early CT Score 


\section{Introduction}

71 Stroke-associated pneumonia (SAP) is one of the most frequent complications following 72 stroke, and it affects about $7 \%$ to $31.3 \%$ of all stroke patients (Chinese Expert Consensus Group 73 on Diagnosis and Treatment of Stroke-associated Pneumonia, 2010; Chumbler et al., 2010;

74 Harms et al., 2013; Nam et al., 2018; Singer., 2009). Evidence shows that SAP has a bad impact 75 on patient outcome, lengthens the duration of hospitalization, and even increases long-term 76 disability or mortality (Alberti et al., 2011; Hilker et., 2003). There are several risk factors that relate to the development of pneumonia after stroke, all of which can be roughly grouped into two categories: the basic condition of the patient (e.g., age, past medical history) and the stroke incident itself (e.g., stroke severity or deficits such as conscious disturbance, dysphagia, and immunosuppression induced by stroke). Although there has been some progress in the treatment of SAP, early identification of patients of stroke with high risk of pneumonia might help to provide preventive measures and reduce the incidence of SAP (Kalra et al., 2015; Meisel et al., 2015; Westendorp et al., 2015).

4 Due to the lack of special clinical manifestations for the SAP in early stage, the pneumonia of these patients tends to be more serious when the SAP is diagnosed, which brings great challenges with regard to treatment. Several risk-scoring models have been proposed to help in the identification of patients at high risk of SAP (Harms et al., 2013; Hoffmann et al., 2013; Ji et al., 2013; Kwon et al., 2006). These predictive models are mostly based on clinical findings, but the clinical manifestations of SAP are frequently atypical and vague (Kishore et al., 2015; Li et al., 2014). Diffusion-Weighted Imaging-Alberta Stroke Program Early CT Score (DWIASPECTS), which comes from Alberta Stroke Program Early CT Score (ASPECTS), is simple and reliable and identifies stroke patients who are unlikely to make an independent recovery despite thrombolytic treatment (Barber et al., 2000; Morita et al., 2009). Researchers have adopted ASPECTS to evaluate the relationship between stroke localization and stroke-associated infection (SAI) (Morita et al., 2009). However, there is a lack of further study to confirm this value of predicting stroke-associated pneumonia for patients with acute ischemic stroke (AIS). The aim of this study was to assess the value of DWI-ASPECTS in predicting SAP for patients with AIS. 


\section{Materials \& Methods}

\section{Patients and population}

104

105

106

107

108

109

110

111

112

113

114

115

116

117

118

119

120

121

122

123

124

125

126

127

128

129

130

131

132

133

134

135

136

137

138

139

140

The retrospective study identified AIS patients $(\mathrm{n}=809)$ who suffered stroke for the first time in Renmin Hospital of Wuhan University between August 2017 and August 2019. We enrolled 291 AIS patients based on the inclusion criteria, which were: patients with AIS who were admitted to hospital within 3 days of onset, AIS diagnosed based on the WHO criteria, and AIS confirmed by brain magnetic resonance image (MRI). The exclusion criteria were: a length of hospital stay $<72$ hours or the onset time of AIS $>72$ hours before admission $(n=243)$; diseases such as severe liver and kidney dysfunction, heart failure, tumour, and blood system and autoimmune diseases $(n=64)$; signs, symptoms, or reported suffering, at the time of admission, of chronic obstructive lung disease (COPD), asthma, or other pulmonary diseases $(\mathrm{n}=61)$; a lack of diffusion-weighted image (DWI) sequence in MRI or no acute infarct on the MRI ( $\mathrm{n}=54)$; National Institutes of Health Stroke Scale (NIHSS) score $=0(n=96)$ (Figure 1).

\section{Data collection and clinical assessment}

The study protocol was approved by the ethics committee of Renmin Hospital of Wuhan University (2017K-C043).Informed consent, written or verbal, was obtained from all participants. The clinical data collection included prior medical history (hypertension, diabetes mellitus, and atrial fibrillation), result of routine blood test, partial blood biochemical examination (levels of glucose, lipids, albumin, homocysteine, C-reactive protein, serum amyloid A), head MRI, chest X-ray photographs or pulmonary CT, and NIHSS at admission. The neutrophil-to-lymphocyte ratio (NLR), known as a systemic inflammation and infection mark, has shown an excellent power for predicting pneumonia (Curbelo et al., 2017; Lee et al., 2016; Nam et al., 2009). Thus, we also evaluated the impact of NLR, as well as neutrophil-tomonocyte ratio (NMR), on patients with SAP. The NLR and NMR were calculated by dividing the absolute neutrophil count by the absolute lymphocyte and monocyte counts, respectively.

In this study, SAP was diagnosed according to the criteria of the Chinese Expert Consensus on Diagnosis and Treatment of Stroke-Associated Pneumonia (Chinese Expert Consensus Group on Diagnosis and Treatment of Stroke-associated Pneumonia, 2010). It included newly emerging or progressively infiltrating pulmonary lesions in AIS patients' chest images combined with more than two of the following clinical manifestations of infection: 1) body temperatures exceeding $38^{\circ} \mathrm{C}$ (rule out other causes of fever); 2) new respiratory symptoms such as cough and sputum, or pre-existing respiratory disease symptoms with or without chest pain or respiratory rate more than $25 / \mathrm{min}$; 3 ) signs of pulmonary consolidation (percussion test presented with dull sound, enhanced vocal fremitus in palpation and/or moist rales in auscultation; 4) Peripheral blood white blood cell count $\geq 10 \times 10^{9} / \mathrm{L}$ or $\leq 4 \times 10^{9} / \mathrm{L}$ with or without left shift of nucleus. Some pulmonary diseases (tuberculosis, pulmonary tumour, non-infective interstitial lung disease, pulmonary oedema, pulmonary embolism, and pulmonary atelectasis), which might be similar to pneumonia with respect to clinical manifestations, were excluded. 
141

142

143

144

145

146

147

148

149

150

151

152

153

154

155

156

157

158

159

160

161

162

163

164

165

166

167

168

169

170

171

172

173

174

175

176

177

178

179

Patients with acute ischemic stroke were arranged to complete a MRI examination within 24 hours of their admission. The DWI-ASPECTS score was calculated at the start of the study based on the scoring rules which described in this manuscript. For anterior circulation stroke, we referred to the assessment method of Singer: the score calculated by the 10 regions shown in Figure 2 (Singer et al., 2009). We subtracted 1 point for the area of early ischemic change for each of the defined regions. For posterior circulation stroke, we referred to the assessment method of Puetz, following the rule of subtracting 1 point for the regions of left or right thalamus, cerebellum, or PCA-territory, respectively; and subtracting 2 points for the regions of any part of the midbrain or pons (Puetz et al., 2008). A DWI-ASPECTS of 10 points meant no early ischemic change shown in the DWI scan; a 0 score implied ischemic involvement throughout the anterior circulation or the posterior circulation territory.

\section{Statistical analysis}

Data were analysed using SPSS statistics 22.0 for windows (SPSS Inc., Chicago, IL). The measurement data with normal distribution were presented as mean \pm standard deviation (SD), and median with interquartile range (IQR) were used for non-normal distribution. Continuous variables were analysed with Student's t-test or Mann-Whitney U-test, and categorical variables were analysed with Chi-square tests.

Factors with $\mathrm{P}<0.10$ in the univariate analysis were entered into the multivariate analysis. Variables of neutrophil and monocyte, NLR and MNR, WBC were entered into three multivariate logistic regression models separately, because of the high correlation with each other in univariate analysis.

Receiver operating characteristic (ROC) curve analyses were performed using MedCalc Version 15.8.0.0 (Frank Schoonjans, Mariakerke, Belgium). Accuracy in predicting outcome measures was assessed by calculating the area under ROC curve. Comparison of the areas under ROC curves (AUC) was performed using MedCalc Version 15.8.0.0. Correlations were analyzed using Spearman's correlations. All hypotheses were 2-tailed, and a P-value of $<0.05$ was considered significant.

\section{Results}

Among the 291 patients, 74 (25.4\%) subjects were diagnosed with SAP. The baseline characteristics between groups with and without SAP are presented in Table 1. An older age and a higher rate of atrial fibrillation (AF) presented in the SAP group. The SAP group also had higher initial NIHSS scores, WBC, neutrophil counts, levels of CRP, and SAA, while lower platelet (PLT) counts and levels of albumin (ALB) than those in the non-SAP group. What's more, the SAP group had a significantly lower DWI-ASPECTS than the non-SAP group (5 [3-7] versus 7 [6-8]; $\mathrm{P}<0.01$ ). In the SAP group, 15 patients had posterior circulation infarction and 59 anterior circulation infarction (Table 2). There was no significant difference in NIHSS between the groups of posterior circulation infarction and anterior circulation infarction. 
180

181

182

183

184

185

186

187

188

189

190

191

192

193

194

195

196

197

198

199

200

201

202

203

204

205

206

207

208

209

210

211

212

213

214

215

216

217

218

219

The results of the multivariable logistic regression analysis was presented in Table 3. After adjusting for confounders, the difference of DWI-ASPECTS (adjusted odds ratio [aOR] = 1.438; 95\% confidence interval [CI], 1.158-1.787; $\mathrm{P}<0.01)$ remained significant. Furthermore, age $(\mathrm{aOR}=0.950 ; 95 \% \mathrm{CI}, 0.918-0.984 ; \mathrm{P}<0.01)$, AF $(\mathrm{aOR}=0.193 ; 95 \% \mathrm{CI}, 0.071-0.526 ; \mathrm{P}<$ $0.01)$, and NIHSS score $(\mathrm{aOR}=0.891 ; 95 \% \mathrm{CI}, 0.830-0.956 ; \mathrm{P}<0.01)$ were also significant, aside from the altered DWI-ASPECTS. The results remained identical after replacing WBC or NLR and NMR with the neutrophil and lymphocyte counts as a sensitivity analysis.

From the ROC analysis, we found that the predictive ability of DWI-ASPECTS (AUC= $0.743>0.7,95 \%$ CI $0.678-0.800$ ) had acceptable discriminatory abilities. The optimal cut-off value was 6 for DWI-ASPECTS, whose sensitivity was $74.32 \%$, specificity was $70.51 \%$, positive predictive value (PPV) was $46.22 \%$, and negative predictive value (NPV) was $88.95 \%$. Other independent risk factors (age, NIHSS, and AF) of SAP in our study were analyzed to investigate the different powers of predictive performance (Figure 3). Although NIHSS (AUC= $0.778,95 \%$ CI $0.716-0.832$ ) showed the highest AUC out of all the independent risk factors referred above, there was no significant difference between NIHSS and DWI-ASPECTS (P > 0.05). We further calculated the AUC which combined DWI-ASPECTS, age, NIHSS, and AF, and found that the AUC was significantly greater than age, NIHSS, AF, and DWI-ASPECTS alone for both outcomes (all $\mathrm{p}<0.01$ ) in either case (Table 4).

This study further explored the correlation between DWI-ASPECTS and the immune status of AIS patients. Although the correlation was not strong, there was significant negative correlation with WBC $(\mathrm{r}=-0.21, \mathrm{p}<0.01)$, Neu $(\mathrm{r}=-0.26, \mathrm{p}<0.01)$, Mon $(\mathrm{r}=-0.17, \mathrm{P}<0.05)$, $\operatorname{NLR}(\mathrm{r}=-0.12, \mathrm{p}<0.05), \operatorname{NMR}(\mathrm{r}=-0.30, \mathrm{p}<0.01)$ and SAA $(\mathrm{r}=-0.18, \mathrm{p}<0.05)$, and a significant positive correlation with lymphocyte counts $(\mathrm{r}=0.19, \mathrm{p}<0.01)$ and PLT $(\mathrm{r}=0.18, \mathrm{P}$ $<0.01$ ), shown in figure 4 .

\section{Discussion}

Early and accurate diagnosis of SAP was regarded as necessary for timely and effective treatment. Originally, ASPECTS and posterior circulation Acute Stroke Prognosis Early CT Score (pc-ASPECTS) were used to identify the early ischemic change in the region of anterior circulation and posterior circulation, respectively. Harms employed ASPECTS graded stroke localization to investigate ischemic lesion characteristics (size, localization) correlation with immune competence (monocytic human leukocyte antigen-DR [HLA-DR] expression) and poststroke infections (Harms et al., 2011). Thus, considering it is more conclusive to find the stroke localization within a short time by DWI sequence in MRI (Sugimori et al., 2012), this study adopted DWI-ASPECTS to explore the relationship with SAP and test the value in predicting the risk of SAP for AIS patients. The results suggested that DWI-ASPECTS has a good predictive value for the diagnosis of SAP and shows an excellent predictive ability when combined with age, AF, and NIHSS.

NIHSS was a 15-item impairment scale which recommended as a valid tool to assess stroke severity (Kasner et al., 2006), and shown as good predictive value as DWI-ASPECTS for the 
220

221

222

223

224

225

226

227

228

229

230

231

232

233

234

235

236

237

238

239

240

241

242

243

244

245

246

247

248

249

250

251

252

253

254

255

256

257

258

259

diagnosis of SAP in this study. To reduce the detection bias, clinicians needed to be trained in how to accurate evaluation of NIHSS (Lyden et al., 2005). In contrast, the evaluation method of DWI-ASPECTS which calculated on the basis of special neuroanatomical area seemed to be more convenient. Assessors could follow the neuroanatomical areas to score objectively, when the DWI sequence in MRI was completed. However, it was undeniable that NIHSS has an important clinical application in predicting the outcome for stroke patient.

As we all know, there are various factors (consciousness disturbance, dysphagia, aspiration, over-activation of the sympathetic nerve system, stroke-induced immunodepression, etc.) that contribute to the occurrence of SAP. What's more, several studies have reported that special anatomy of ischemic lesions and infarct volume, which is associated with systemic immunodepression, might contribute to the high risk of post-stroke infection as well (Urra et al., 2017). The semiquantitative scoring system of DWI-ASPECTS was calculated on the basis of special neuroanatomical area and reflected the volume of infarct to a certain degree. In the course of investigating the possible mechanism that DWI-ASPECTS might be a predictor of SAP, the magnitude of the DWI-ASPECTS was associated with peripheral immune changes by doing the correlation analysis between DWI-ASPECTS and peripheral blood inflammatory cells.

Stroke-induced immunosuppression is an important risk factor of SAP has been widely recognized (Chamorro et al., 2012; Hoffmann et al., 2017; Kemmling et al., 2013; Mracsko et al., 2014). A characteristic of stroke-induced immunosuppression is the impairment of immune function, such as a decrease in the number of circulating lymphocytes and deactivation of monocytes. Lymphopenia, in turn, may predispose to the development of infections (MerayoChalico J et al., 2013;Klehmet J et al.,2009). Compared with non-SAP patients in our study, the SAP patients had higher levels of WBC and neutrophil and lower levels of lymphocyte, which was in line with previous studies(Dirnagl U et al., 2007). However, among these factors only DWI-ASPECTS, age, AF, and NIHSS remained significant contributors after adjusting for confounders in the multivariable logistic regression model. Surprisingly, to some extent, lymphopenia didn't remain as independent factor in the multivariable regression model. After consideration, we could give explanations as follows. High initial NIHSS score, DWI-ASPECTS or lymphopenia could be used to represent stroke severity. Lymphopenia not remaining significant was potentially due to interaction effects influenced by many substances and the surrounding microenvironments. In addition (Feng et al., 2018; Nam et al., 2018; Westendorp et al., 2015), a high NLR was reported to show a good correlation with SAP events in patients with AIS. As is shown in Figure 4, these results indirectly suggest that DWI-ASPECTS has relation to immunosuppression, although the correlation was not strong. Of course, these assumptions need more experimental data to validate and more supports from clinical data that excluding the bias caused by the low patient case number.

Besides, aspiration which was likely caused by dysphagia was another independent risk factor of SAP (Chamorro et al., 2012; Hoffmann et al., 2017). It was reported that dysphagia carried a seven fold increased risk of pneumonia after stroke (Singh S et al., 2006). The survivor of brainstem stroke might experience deficits in pharyngeal and upper sophageal sphincter 
260 function, and thus further increase the risk of SAP (Prabhjot K., 2016; Sasegbon A et al., 261 2017). Thus, the management of dysphagia was one of the key issues in preventing the 262 occurrence of aspiration pneumonia in stroke patients (Radhakrishnan S., 2013). Compared with 263 these did not reach brainstem, ischemic lesions reached brainstem in AIS patients exerted no 264 impact on the development of SAP in this study. The probable reason is that more preventive 265 care were given to these AIS patient with brainstem stroke.

266 There were several limitations to this study. Firstly, this study was a retrospective study. 267 Although a relatively large sample size was involved, about one third of the AIS patients who 268 suffered stroke for the first time were included in the analysis. Therefore, a possibility of 269 selection bias inevitably existed. We adopted the Chinese Expert Consensus on Diagnosis and 270 Treatment of Stroke-Associated Pneumonia as diagnostic criteria for SAP as well as Li (Li et al., 271 2014)), and calculated that the incidence rate of SAP was $25.4 \%$, which was in accord with the 272 result of $\mathrm{Li}(24.1 \%)$. The change in immune conditions between non-SAP and SAP patients 273 concur with the opinion of stroke-induced immunosuppression. Taking these results, which align 274 with previous research, into account, we believed that this research approach was reasonable and 275 reliable. Secondly, the record of dysphagia screening was incomplete in this retrospective study, 276 which might be a drawback. At the same time, there was a lack of specific recommendations on 277 the standardized criteria for dysphagia screening in international stroke guidelines. Thirdly, 278 DWI-ASPECTS based on the diffusion-weighted image of MRI was simpler and more sensitive 279 than CT scan in finding the EIC, and it showed a strong predictive ability of SAP occurrence for 280 AIS patients. But before generalization of the findings to clinical fields, a prospective, 281 multicentre study was needed.

282

283

\section{Conclusions}

284 In conclusion, DWI-ASPECTS has a good predictive value for the diagnosis of SAP.

285 Because the DWI-ASPECTS can be easily calculated from the DWI sequence in MRI

286

287 examination, it may help to select high-risk patients to begin intervention in time. However, to prove its validity further, DWI-ASPECTS needs to be assessed prospectively and at multiple 288 centres.

\section{Funding}

290 This work was supported by National Natural Science Foundation of China grant (No.

29181770089 ,

292 81970082).

\section{Acknowledgements}

294 We declare no conflicts of interest.

295

\section{References}


297

298

299

300

301

302

303

304

305

306

307

308

309

310

311

312

313

314

315

316

317

318

319

320

321

322

323

324

325

326

327

328

329

330

331

332

333

334

335

336

337

338

Alberti A, Agnelli G, Caso V, Venti M, Acciarresi M, D'Amore C, et al. Non-neurological complications of acute stroke: frequency and influence on clinical outcome. Intern Emerg Med 2011;6 Suppl 1:119-123.

Barber PA, Demchuk AM, Zhang J, Buchan AM. Validity and reliability of a quantitative computed tomography score in predicting outcome of hyperacute stroke before thrombolytic therapy. Lancet 2000;355:1670-1674.

Chinese Expert Consensus Group on Diagnosis and Treatment of Stroke-associated Pneumonia. Chinese expert consensus on diagnosis and treatment of stroke-associated pneumonia. Chin J Inter Med 2010; 49:1075-1078.

Chamorro A, Meisel A, Planas AM, Urra X, van de Beek D, Veltkamp R. The immunology of acute stroke. Nat Rev Neurol 2012;8:401-410.

Chumbler NR, Williams LS, Wells CK, Lo AC, Nadeau S, Peixoto AJ, et al. Derivation and validation of a clinical system for predicting pneumonia in acute stroke. Neuroepidemiology 2010; 34:193-199.

Curbelo J, Luquero Bueno S, Galvan-Roman JM, Ortega-Gomez M, Rajas O, FernandezJimenez G, et al. Inflammation biomarkers in blood as mortality predictors in communityacquired pneumonia admitted patients: Importance of comparison with neutrophil count percentage or neutrophil-lymphocyte ratio. PLoS One 2017;12:e0173947.

Dirnagl U, Klehmet J, Braun JS, Harms H, Meisel C, Ziemssen T, et al. Stroke-induced immunodepression: experimental evidence and clinical relevance. Stroke 2007,38(2 Suppl):770-773.

Feng HX, Cheng Y, Zhu W, Jiang LL, Dong XF, Gui Q, et al. T-lymphocyte subsets as a predictive biomarker for stroke-associated pneumonia. Am J Transl Res 2018;10:43674375.

Harms H, Reimnitz P, Bohner G, Werich T, Klingebiel R, Meisel C, et al. Influence of stroke localization on autonomic activation, immunodepression, and post-stroke infection. Cerebrovasc Dis 2011;32(6):552-560.

Harms H, Grittner U, Droge H, Meisel A. Predicting post-stroke pneumonia: the PANTHERIS score. Acta Neurol Scand 2013;128:178-184.

Hilker R, Poetter C, Findeisen N, Sobesky J, Jacobs A, Neveling M, et al. Nosocomial pneumonia after acute stroke: implications for neurological intensive care medicine. Stroke 2003;34:975-981.

Hoffmann S, Malzahn U, Harms H, Koennecke HC, Berger K, Kalic M, et al. Development of a clinical score (A2DS2) to predict pneumonia in acute ischemic stroke. Stroke 2012;43:2617-2623.

Hoffmann S, Harms H, Ulm L, Nabavi DG, Mackert BM, Schmehl I, et al. Stroke-induced immunodepression and dysphagia independently predict stroke-associated pneumonia - The PREDICT study. J Cereb Blood Flow Metab 2017;37:3671-3682.

Ji R, Shen H, Pan Y, Wang P, Liu G, Wang Y, et al. Novel risk score to predict pneumonia after acute ischemic stroke. Stroke 2013;44:1303-1309.

Kalra L, Irshad S, Hodsoll J, Simpson M, Gulliford M, Smithard D, et al. Prophylactic antibiotics after acute stroke for reducing pneumonia in patients with dysphagia (STROKE-INF): a 
339

340

341

342

343

344

345

346

347

348

349

350

351

352

353

354

355

356

357

358

359

360

361

362

363

364

365

366

367

368

369

370

371

372

373

374

375

376

prospective, cluster-randomised, open-label, masked endpoint, controlled clinical trial. Lancet 2015;386:1835-1844.

Kasner SE. Clinical interpretation and use of stroke scales. Lancet Neurol 2006;5(7):603-612. Kemmling A, Lev MH, Payabvash S, Betensky RA, Qian J, Masrur S, et al. Hospital acquired pneumonia is linked to right hemispheric peri-insular stroke. PLoS One 2013;8:e71141.

Kishore AK, Vail A, Chamorro A, Garau J, Hopkins SJ, Di Napoli M, et al. How is pneumonia diagnosed in clinical stroke research? A systematic review and meta-analysis. Stroke 2015;46:1202-1209.

Klehmet J, Harms H, Richter M, Prass K, Volk HD, Dirnagl U, et al. Stroke-induced immunodepression and post-stroke infections: lessons from the preventive antibacterial therapy in stroke trial. Neuroscience 2009,158(3):1184-1193.

Kwon HM, Jeong SW, Lee SH, Yoon BW. The pneumonia score: a simple grading scale for prediction of pneumonia after acute stroke. Am J Infect Control 2006;34:64-68.

Lee JH, Song S, Yoon SY, Lim CS, Song JW, Kim HS. Neutrophil to lymphocyte ratio and platelet to lymphocyte ratio as diagnostic markers for pneumonia severity. Br J Biomed Sci 2016;73:140-142.

Li L, Zhang LH, Xu WP, Hu JM: Risk assessment of ischemic stroke associated pneumonia. World J Emerg Med 2014;5:209-213.

Lyden P, Raman R, Liu L, Grotta J, Broderick J, Olson S, et al. NIHSS training and certification using a new digital video disk is reliable. Stroke 2005,36(11):2446-2449.

Meisel A, Smith CJ. Stroke: Preventive antibiotics for stroke-associated pneumonia. Nat Rev Neurol 2015;11:672-673.

Merayo-Chalico J, Gómez-Martín D, Piñeirúa-Menéndez A, Santana-De Anda K, AlcocerVarela J. Lymphopenia as risk factor for development of severe infections in patients with systemic lupus erythematosus: a case-control study. QJM. 2013,106(5):451-457.

Morita N, Harada M, Uno M, Matsubara S, Nagahiro S, Nishitani H. Evaluation of initial diffusion-weighted image findings in acute stroke patients using a semiquantitative score. Magn Reson Med Sci 2009;8:47-53.

Mracsko E, Liesz A, Karcher S, Zorn M, Bari F, Veltkamp R. Differential effects of sympathetic nervous system and hypothalamic-pituitary-adrenal axis on systemic immune cells after severe experimental stroke. Brain Behav Immun 2014;41:200-209.

Nam KW, Kim TJ, Lee JS, Kwon HM, Lee YS, Ko SB, et al. High Neutrophil-to-Lymphocyte Ratio Predicts Stroke-Associated Pneumonia. Stroke 2018;49:1886-1892.

Puetz V, Sylaja PN, Coutts SB, Hill MD, Dzialowski I, Mueller P, et al. Extent of hypoattenuation on CT angiography source images predicts functional outcome in patients with basilar artery occlusion. Stroke 2008;39:2485-2490.

Prabhjot K. Dysphagia in Wallenberg's Syndrome: The Challenge in Treatment. Open Access J Neurol Neurosurg. 2016; 1(3): 002555563. 
377 Radhakrishnan S, Menon UK, Anandakuttan A. A combined approach of bedside clinical

378

379

380

381

382

383

384

385

386

387

388

389

390

391

392

393

394

395 examination and flexible endoscopic evaluation of swallowing in poststroke dysphagia: A pilot study. Ann Indian Acad Neurol. 2013;16(3):388-393.

Sasegbon A, Hamdy S. The anatomy and physiology of normal and abnormal swallowing in oropharyngeal dysphagia. Neurogastroenterol Motil 2017,29(11).

Singer OC, Kurre W, Humpich MC, Lorenz MW, Kastrup A, Liebeskind DS, et al. Risk assessment of symptomatic intracerebral hemorrhage after thrombolysis using DWIASPECTS. Stroke 2009; 40:2743-2748.

Singh S, Hamdy S. Dysphagia in stroke patients. Postgrad Med J 2006,82(968):383-391. Sugimori H, Kanna T, Yamashita K, Kuwashiro T, Yoshiura T, Zaitsu A, et al. Early findings on brain computed tomography and the prognosis of post-cardiac arrest syndrome: application of the score for stroke patients. Resuscitation 2012;83:848-854.

Urra X, Laredo C, Zhao Y, Amaro S, Rudilosso S, Renu A, et al. Neuroanatomical correlates of stroke-associated infection and stroke-induced immunodepression. Brain Behav Immun 2017;60:142-150.

Westendorp WF, Vermeij JD, Zock E, Hooijenga IJ, Kruyt ND, Bosboom HJ, et al. The Preventive Antibiotics in Stroke Study (PASS): a pragmatic randomised open-label masked endpoint clinical trial. Lancet 2015;385:1519-1526. 
$397 \mathrm{COPD}=$ chronic obstructive pulmonary disease; $\mathrm{MRI}=$ magnetic resonance imaging; $\mathrm{DWI}=$ Diffusion-Weighted Imaging; NIHSS = National Institutes of Health Stroke Scale; TIA = transient ischemic attack.

400

401

Figure 2. Diffusion-Weighted Imaging-Alberta Stroke Program Early CT Score (DWI402 ASPECTS).

403

(A) The anterior circulation stroke assessed by DWI-ASPECTS. $\mathrm{C}=$ caudate, $\mathrm{I}=$ insular ribbon, 404

$\mathrm{L}=$ lentiform, $\mathrm{IC}=$ internal capsule, $\mathrm{M} 1=$ anterior portion of the MCA cortex, $\mathrm{M} 2=\mathrm{MCA}$

405 cortex lateral to the insular ribbon, M3 = posterior MCA cortex, M4 = anterior MCA territories, M5 = lateral MCA territories, and M6 = posterior MCA territories. One point was subtracted for the area of early ischemic change for each of the defined regions; (B) The posterior circulation

408

409

410

411

412

413

414

415

416

417

418

419

420

421

422

423

424

425

426

427 stroke assessed by DWI-ASPECTS. From 10 points, 1 or 2 points each (as indicated) were subtracted for early ischemic changes in the left or right thalamus, cerebellum, or PCA territory, respectively ( 1 point); any part of midbrain or pons ( 2 points).

\section{Figure 3. Comparison of area under curve (AUC) between DWI-ASPECTS and other} independence factors in the prediction of SAP by Receiver Operating Characteristic (ROC) curves.

The additive effect of DWI-ASPECTS, AF, Age and NIHSS in the prediction of SAP was calculated also.

Figure 4. The correlation between DWI-ASPECTS and the immune status of patients with AIS.

There was a negative correlation with $\mathrm{WBC}\left(\mathrm{R}^{2}=0.04, \mathrm{P}=0.00\right)$, Neu $\left(\mathrm{R}^{2}=0.08, \mathrm{P}=0.00\right)$, and NLR $\left(\mathrm{R}^{2}=0.02, \mathrm{P}=0.02\right), \mathrm{NMR}\left(\mathrm{R}^{2}=0.09, \mathrm{P}=0.00\right)$ and a positive correlation with lymphocytes $\left(\mathrm{R}^{2}=0.03, \mathrm{p}=0.00\right)$, and PLT $\left(\mathrm{R}^{2}=0.04, \mathrm{P}=0.00\right)$. Abbreviations: AIS = acute ischemic stroke, $\mathrm{WBC}=$ white blood cell count, $\mathrm{Neu}=$ neutrophil count, $\mathrm{Lyn}=$ lymphocyte count, Mon = monocyte count, NLR = neutrophil-to-lymphocyte ratio, NMR = neutrophil-tomonocyte ratio, $\mathrm{PLT}=$ platelet count, $\mathrm{SAA}=$ serum amyloid $\mathrm{A}, \mathrm{CRP}=\mathrm{C}$-reactive protein.

\section{Table 1 Baseline characteristics of the patients}


428 a Continuous variables were expressed as mean \pm standard deviation or as median (interquartile range).

429 Categorical variables were expressed as frequency (percent); ${ }^{\dagger}$ Mann-Whitney U-test, ${ }^{\star}$ Student’s t-test, $§$

$430 \chi^{2}$-test. Abbreviations: NIHSS = National Institutes of Health Stroke Scale, DWI-ASPECTS $=$ Diffusion-

431 Weighted Imaging-Alberta Stroke Program Early CT Score, WBC $=$ White Blood cell Count, RBC $=$ Red

432 Blood cell Count, PLT = Platelet Count, SAA = Serum Amyloid A, CRP = C-Reactive Protein, HCY =

433 Homocysteine.

434

435

Table 2 The characteristics of different ischemic region in SAP patients

436

437 Table 3 Logistic regression of associations between risk factors for SAP

438 Abbreviations: AF = Atrial fibrillation, HDL $=$ High-density lipoprotein, NLR = neutrophil-to-

439 lymphocyte ratio, $\mathrm{NMR}$ = neutrophil-to-monocyte ratio. We brought sex which reported a risk

440 factor of SAP into analysis, although it was shown no significant in this study. And either

441 neutrophils, lymphocytes, NLR, NMR or WBC was not founded to be an significant predictor

442 regardless of model.

$443{ }^{1}$ Model 1: Adjusted with $\mathrm{P}<0.10$ in univariate analysis (Sex, Age, DWI-ASPECTS, NIHSS, 444 AF, Neutrophil, Lymphocyte, PLT, SAA, CRP, ALB, and HDL). ${ }^{2}$ Model 2: Adjusted with P < 4450.10 in univariate analysis (Sex, Age, DWI-ASPECTS, NIHSS, AF, NLR, NMR, PLT, SAA, 446 CRP, ALB, and HDL). ${ }^{3}$ Model 3: Adjusted with $\mathrm{P}<0.10$ in univariate analysis (Sex, Age,

447 DWI-ASPECTS, NIHSS, AF, WBC, PLT, SAA, CRP, ALB, and HDL).

448

449 Table 4 Pairwise comparison of ROC curves

450 Abbreviations: AF $=$ Atrial fibrillation, DWI-ASPECTS $=$ Diffusion Weighted Imaging-Alberta

451 Stroke Program Early CT Score, NIHSS = National Institutes of Health Stroke Scale. AF, age, 452 DWI-ASPECTS and NIHSS are all significant predictors regardless of model. 


\section{Table $\mathbf{1}$ (on next page)}

Baseline characteristics of the patients

${ }^{\text {a }}$ Continuous variables were expressed as mean \pm standard deviation or as median (interquartile range). Categorical variables were expressed as frequency (percent); ${ }^{\dagger}$ MannWhitney U-test, ${ }^{\ddagger}$ Student's t-test, ${ }^{\S} \chi^{2}$-test. Abbreviations: NIHSS $=$ National Institutes of Health Stroke Scale, DWI-ASPECTS = Diffusion Weighted Imaging-Alberta Stroke Program Early CT Score, $\mathrm{WBC}=$ White Blood cell Count, $\mathrm{RBC}=$ Red Blood cell Count, $\mathrm{PLT}=$ Platelet Count, SAA = Serum Amyloid A, CRP = C-Reactive Protein, $\mathrm{HCY}=$ Homocysteine. 
Table 1 Baseline characteristics of the patients

\begin{tabular}{|c|c|c|c|}
\hline Characteristics a & $\operatorname{SAP}(n=74)$ & Non-SAP $(\mathrm{n}=217)$ & $P$-Value \\
\hline Male: Female $\S$ & $47: 27$ & $142: 75$ & 0.764 \\
\hline Age, years $\$$ & $72.32 \pm 13.67$ & $64.19 \pm 12.67$ & 0.000 \\
\hline NIHSS ${ }^{\dagger}$ & $12(5-16)$ & $4(2-7)$ & 0.000 \\
\hline DWI-ASPECTS ${ }^{\dagger}$ & $5(3-7)$ & $7(6-8)$ & 0.000 \\
\hline Hypertension, n (\%) § & $47(63.5)$ & $137(63.1)$ & 0.953 \\
\hline Diabetes, $n(\%) \S$ & $17(23.0)$ & $71(32.7)$ & 0.115 \\
\hline Atrial fibrillation, $\mathrm{n}(\%) \S$ & $17(23.0)$ & $22(10.1)$ & 0.005 \\
\hline Neutrophil $\left(\times 10^{9} / \mathrm{L}\right)^{\dagger}$ & $6.08(4.01-9.03)$ & $4.72(3.54-6.32)$ & 0.001 \\
\hline Monocyte $\left(\times 10^{9} / \mathrm{L}\right)^{\dagger}$ & $0.55(0.42-0.73)$ & $0.51(0.41-0.68)$ & 0.298 \\
\hline Lymphocyte $\left(\times 10^{9} / \mathrm{L}\right)^{\dagger}$ & $1.43(0.85-1.79)$ & $1.55(1.22-2.02)$ & 0.017 \\
\hline WBC $\left(\times 10^{9} / \mathrm{L}\right)$ & $8.70 \pm 3.25$ & $7.50 \pm 2.36$ & 0.005 \\
\hline $\mathrm{RBC}\left(\times 10^{12} / \mathrm{L}\right)$ & $4.47 \pm 0.68$ & $4.59 \pm 0.62$ & 0.159 \\
\hline $\operatorname{PLT}\left(\times 10^{9} / \mathrm{L}\right) *$ & $193.21 \pm 75.00$ & $214.64 \pm 66.93$ & 0.024 \\
\hline $\mathrm{SAA}(\mathrm{mg} / \mathrm{L})^{\dagger}$ & $10.06(5-52.51)$ & $5.07(5.00-9.76)$ & 0.002 \\
\hline $\mathrm{CRP}(\mathrm{mg} / \mathrm{L})^{\dagger}$ & $5.06(1.62-15.02)$ & $2.13(0.73-5.86)$ & 0.001 \\
\hline $\operatorname{Albumin}(\mathrm{g} / \mathrm{L})^{\ddagger}$ & $39.97 \pm 4.65$ & $41.49 \pm 3.75$ & 0.006 \\
\hline Blood glucose $(\mathrm{mmol} / \mathrm{L}) \dagger$ & $6.29(5.20-7.80)$ & $5.64(4.80-7.21)$ & 0.076 \\
\hline Triglyceride $(\mathrm{mmol} / \mathrm{L})^{\dagger}$ & $1.2(0.87-1.73)$ & $1.37(1.03-2.03)$ & 0.079 \\
\hline High-density lipoprotein & $1.1(0.88-1.39)$ & $1.03(0.85-1.25)$ & 0.054 \\
\hline Low-density lipoprotein & $2.28(1.82-3.06)$ & $2.55(2.05-3.08)$ & 0.123 \\
\hline $\mathrm{HCY}(\mu \mathrm{mol} / \mathrm{L})^{\dagger}$ & $17.33(12.74-22.60)$ & $15.41(12.91-19.65)$ & 0.487 \\
\hline Ischemia region $\S$ & & & 0.532 \\
\hline Anterior circulation, n (\%) & $59(24.70)$ & $180(75.30)$ & \\
\hline Posterior circulation, $\mathrm{n}(\%)$ & $15(28.80)$ & $37(71.20)$ & \\
\hline Brain stem infarcts, $\mathrm{n}(\%) \S$ & $11(14.9)$ & $24(11.1)$ & 0.385 \\
\hline
\end{tabular}




\section{Table 2 (on next page)}

The characteristics of different ischemic region in SAP patients

${ }^{\dagger}$ Continuous variables were expressed as media (interquartile range). Mann-Whitney U-test was used for statistical analysis. 
1

Table 2. The characteristics of different ischemic region in SAP patients

\begin{tabular}{cccc}
\hline & $\begin{array}{c}\text { Anterior circulation } \\
(\mathrm{n}=59)\end{array}$ & $\begin{array}{c}\text { Posterior circulation } \\
(\mathrm{n}=15)\end{array}$ & $\mathrm{P}$ \\
\hline DWI-ASPECTS- $^{+}$ & $5(3-6)$ & $7(5-8)$ & 0.014 \\
NIHSS $^{\dagger}$ & $13(7-16)$ & $8(3-20)$ & 0.215 \\
\hline
\end{tabular}

2

3

4

5

6

7

8

9

10

11

12

13 
14

Table 2. The characteristics of different ischemic region in SAP patients

\begin{tabular}{cccc}
\hline & $\frac{\text { Anterior circulation }}{(\mathrm{n}=59)}$ & $\frac{\text { Posterior circulation }}{(\mathrm{n}=15)}$ & $\underline{\mathrm{P}}$ \\
\hline$\underline{\text { NIHSS }{ }^{\dagger}}$ & $\underline{13(7-16)}$ & $\underline{8(3-20)}$ & $\underline{0.215}$ \\
\hline
\end{tabular}




\section{Table 3 (on next page)}

Logistic regression of associations between risk factors for SAP

Abbreviations: $\mathrm{AF}=$ Atrial fibrillation, $\mathrm{HDL}=$ High-density lipoprotein, NLR $=$ neutrophil-tolymphocyte ratio, NMR = neutrophil-to-monocyte ratio. We brought sex which reported a risk factor of SAP into analysis, although it was shown no significant in this study.Model 1:

Adjusted with $\mathrm{P}<0.10$ in univariate analysis (Sex, Age, DWI-ASPECTS, NIHSS, AF, Neutrophil, Lymphocyte, PLT, SAA, CRP, ALB, and HDL). Model 2: Adjusted with $\mathrm{P}<0.10$ in univariate analysis (Sex, Age, DWI-ASPECTS, NIHSS, AF, NLR, NMR, PLT, SAA, CRP, ALB, and HDL).Model 3: Adjusted with $P<0.10$ in univariate analysis (Sex, Age, DWI-ASPECTS, NIHSS, AF, WBC, PLT, SAA, CRP, ALB, and HDL). 
Table 3 Logistic regression of associations between risk factors for SAP

\begin{tabular}{|c|c|c|c|c|c|c|}
\hline \multirow[b]{2}{*}{ Parameters } & \multicolumn{6}{|c|}{ Model 1} \\
\hline & B & $\mathrm{SE}$ & Wald & $\mathrm{P}$ & OR & $95 \% \mathrm{CI}$ \\
\hline Sex & -0.231 & 0.444 & 0.271 & 0.603 & 0.794 & $0.332-1.895$ \\
\hline Age & -0.051 & 0.018 & 8.259 & 0.004 & 0.950 & $0.918-0.984$ \\
\hline DWI-ASPECTS & 0.363 & 0.111 & 10.765 & 0.001 & 1.438 & $1.158-1.787$ \\
\hline NIHSS & -0.116 & 0.036 & 10.269 & 0.001 & 0.891 & $0.830-0.956$ \\
\hline $\mathrm{AF}$ & -1.647 & 0.512 & 10.337 & 0.001 & 0.193 & $0.071-0.526$ \\
\hline${ }_{-}^{1}$ Neutrophil & -0.053 & 0.089 & 0.359 & 0.54 & 0.948 & $0.797-1.128$ \\
\hline${ }^{1}$ Lymphocyte & 0.157 & 0.339 & 0.214 & 0.64 & 1.170 & $0.602-2.271$ \\
\hline${ }^{2} \mathrm{NLR}$ & $\frac{-0.009}{-}$ & $\frac{0.036}{-}$ & 0.066 & $\frac{0.79}{-}$ & $\frac{0.991}{-}$ & $\underline{0.922-1.064-}$ \\
\hline${ }_{-}^{2} \mathrm{NMR}$ & $\frac{-0.021-}{-}$ & $\frac{0.061-}{-}$ & 0.117 & $\underline{0.73}$ & $\frac{0.980}{-}$ & $0.870-$ \\
\hline$\underline{3} \mathrm{WBC}$ & $\frac{-0.030}{-}$ & $\frac{0.087-}{-}$ & 0.118 & $\underline{0.73}$ & $\frac{.971}{-}$ & $\underline{0.818-1.151-}$ \\
\hline PLT & 0.001 & 0.003 & 0.106 & 0.745 & 1.001 & $0.995-1.007$ \\
\hline SAA & -0.003 & 0.004 & 0.609 & 0.43 & 0.997 & $0.990-1.004$ \\
\hline CRP & -0.017 & 0.011 & 2.629 & 0.10 & 0.983 & $0.962-1.004$ \\
\hline ALB & 0.094 & 0.051 & 3.467 & 0.063 & 1.099 & $0.995-1.214$ \\
\hline R1and aurar & $-\cap \cap 52$ & $n \cap 60$ & n 570 & $\cap \Delta 47$ & $\cap \mathrm{Q} \Delta \mathrm{O}$ & ก 878-1 ก87 \\
\hline
\end{tabular}


Table 3 Logistic regression of associations between risk factors for SAP

\begin{tabular}{lcccccc}
\hline & $\mathrm{B}$ & $\mathrm{SE}$ & Wald & $\mathrm{P}$ & OR & $95 \% \mathrm{CI}$ \\
\hline Sex & -0.231 & 0.444 & 0.271 & 0.603 & 0.794 & $0.332-1.895$ \\
Age & -0.051 & 0.018 & 8.259 & 0.004 & 0.950 & $0.918-0.984$ \\
DWI-ASPECTS & 0.363 & 0.111 & 10.765 & 0.001 & 1.438 & $1.158-1.787$ \\
NIHSS & -0.116 & 0.036 & 10.269 & 0.001 & 0.891 & $0.830-0.956$ \\
AF & -1.647 & 0.512 & 10.337 & 0.001 & 0.193 & $0.071-0.526$ \\
${ }^{1}$ Neutrophil & -0.053 & 0.089 & 0.359 & 0.549 & 0.948 & $0.797-1.128$ \\
${ }^{1}$ Lymphocyte & 0.157 & 0.339 & 0.214 & 0.644 & 1.170 & $0.602-2.271$ \\
${ }^{2}$ NLR & -0.009 & 0.036 & 0.066 & 0.797 & 0.991 & $0.922-1.064$ \\
${ }^{2}$ NMR & -0.021 & 0.061 & 0.117 & 0.733 & 0.980 & $0.870-1.103$ \\
${ }^{3}$ WBC & -0.030 & 0.087 & 0.118 & 0.731 & 0.971 & $0.818-1.151$ \\
PLT & 0.001 & 0.003 & 0.106 & 0.745 & 1.001 & $0.995-1.007$ \\
SAA & -0.003 & 0.004 & 0.609 & 0.435 & 0.997 & $0.990-1.004$ \\
CRP & -0.017 & 0.011 & 2.629 & 0.105 & 0.983 & $0.962-1.004$ \\
ALB & 0.094 & 0.051 & 3.467 & 0.063 & 1.099 & $0.995-1.214$ \\
Blood sugar & -0.053 & 0.069 & 0.579 & 0.447 & 0.949 & $0.828-1.087$ \\
Triglyceride & 0.055 & 0.230 & 0.058 & 0.810 & 1.057 & $0.673-1.660$ \\
HDL & -0.942 & 0.580 & 2.643 & 0.104 & 0.390 & $0.125-1.214$ \\
\hline
\end{tabular}


Table 4 (on next page)

Pairwise comparison of ROC curves 
Table 4 Pairwise comparison of ROC curves

Table 4 Pairwise comparison of ROC curves

\begin{tabular}{|c|c|c|c|c|c|c|}
\hline Parameters & & Difference between areas & Standard Error & $\begin{array}{c}95 \% \text { Confidence } \\
\text { interval }\end{array}$ & $\mathrm{Z}$ statistic & $P$-Value \\
\hline \multirow{2}{*}{$\mathrm{AF}$} & model_1 & 0.299 & 0.0379 & $0.225-0.373$ & 7.900 & 0.000 \\
\hline & model_3 & 0.297 & 0.0380 & $0.222-0.372$ & 7.811 & 0.000 \\
\hline \multirow[t]{2}{*}{ Age } & model_2 & 0.218 & 0.0447 & $0.130-0.305$ & 4.867 & 0.000 \\
\hline & model_3 & 0.216 & 0.0448 & $0.128-0.304$ & 4.815 & 0.000 \\
\hline DWI_ASPECTS & model_1 & 0.135 & 0.0372 & $0.0621-0.208$ & 3.629 & 0.000 \\
\hline \multirow{3}{*}{ NIHSS } & model_1 & 0.0995 & 0.0320 & $0.0368-0.162$ & 3.111 & 0.002 \\
\hline & model_2 & 0.0992 & 0.0320 & $0.0366-0.162$ & 3.106 & 0.002 \\
\hline & model_3 & 0.0974 & 0.0320 & $0.0347-0.160$ & 3.044 & 0.002 \\
\hline \multicolumn{2}{|c|}{ DWI_ASPECTS $\sim$ NIHSS } & 0.0356 & 0.0385 & $-0.0398-0.111$ & 0.925 & 0.355 \\
\hline
\end{tabular}


Figure 1 (on next page)

Patient Flow-Chart

COPD = Chronic Obstructive Pulmonary Disease; $\mathrm{MRI}=$ Magnetic Resonance Imaging; $\mathrm{DWI}=$ Diffusion-Weighted Imaging; NIHSS = National Institutes of Health Stroke Scale; TIA = Transient Ischemic Attack. 
Figure 1. Patient Flow-Chart.

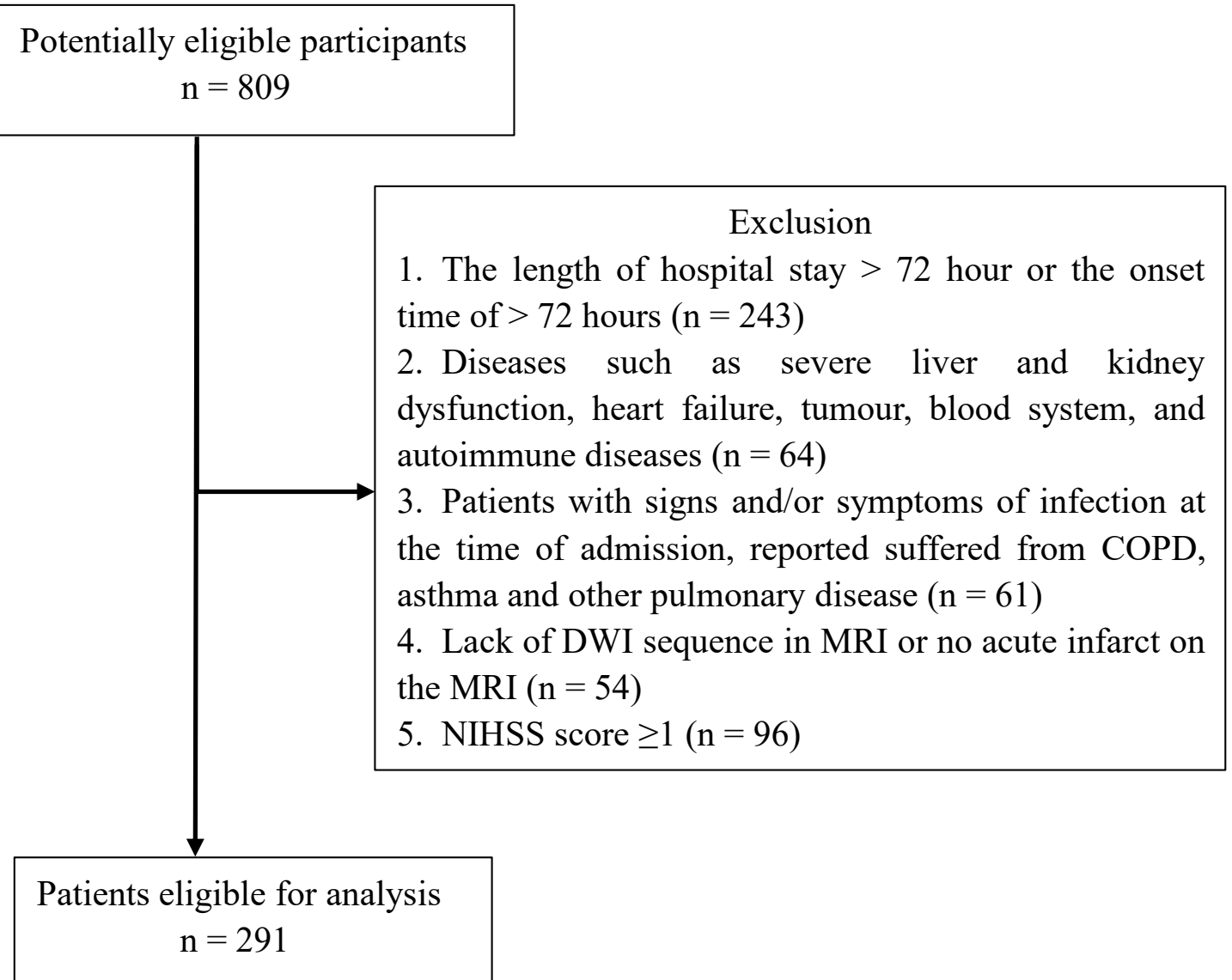




\section{Figure 2 (on next page)}

Diffusion-Weighted Imaging-Alberta Stroke Program Early CT Score (DWI-ASPECTS)

(A) The anterior circulation stroke assessed by DWI-ASPECTS. C = caudate, I = insular ribbon,

$\mathrm{L}=$ lentiform,$I C=$ internal capsule, $M 1=$ anterior portion of the MCA cortex, $M 2=M C A$ cortex lateral to the insular ribbon, M3 = posterior MCA cortex, M4 = anterior MCA territories, M5 = lateral MCA territories, and M6 = posterior MCA territories. One point was subtracted for the area of early ischemic change for each of the defined regions; (B) The posterior circulation stroke assessed by DWI-ASPECTS. From 10 points, 1 or 2 points each (as indicated) were subtracted for early ischemic changes in the left or right thalamus, cerebellum, or PCA territory, respectively (1 point); any part of midbrain or pons (2 points). 
Figure 2. Diffusion-Weighted Imaging-Alberta Stroke Program Early CT Score (DWI-ASPECTS).

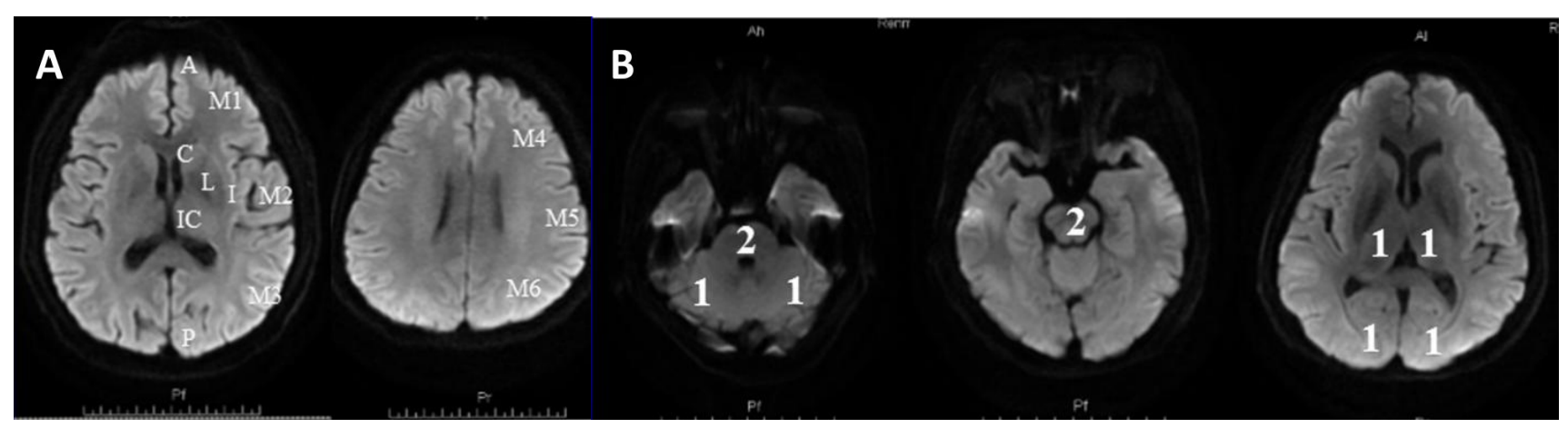


Figure 3 (on next page)

Comparison of area under curve (AUC) between DWI-ASPECTS and other independence factors in the prediction of SAP by Receiver Operating Characteristic (ROC) curves

The additive effect of DWI-ASPECTS, AF, Age and NIHSS in the prediction of SAP was calculated also. 
Figure 3. Comparison of area under curve (AUC) between DWI-ASPECTS and other independence factors in the prediction of SAP by Receiver Operating Characteristic (ROC) curves.
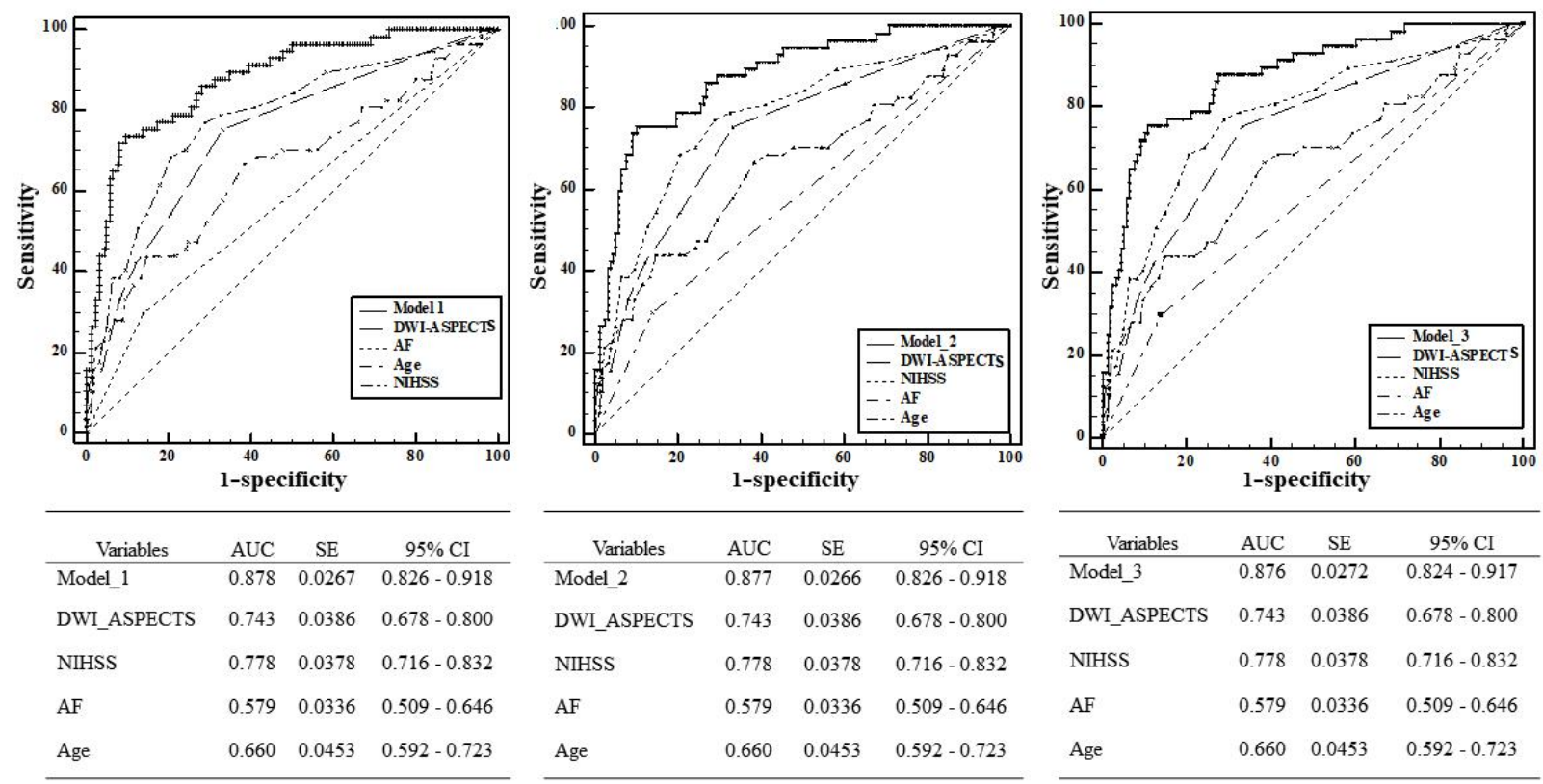
Figure 4 (on next page)

The correlation between DWI-ASPECTS and the immune status of patients with AIS

AIS $=$ acute ischemic stroke, WBC $=$ white blood cell count, $\mathrm{Neu}=$ neutrophil count, Lyn $=$ lymphocyte count, Mon = monocyte count, NLR = neutrophil-to-lymphocyte ratio, NMR = neutrophil-to-monocyte ratio, $\mathrm{PLT}=$ platelet count, $\mathrm{SAA}=$ serum amyloid $\mathrm{A}, \mathrm{CRP}=\mathrm{C}$-reactive protein. 
Figure 4. The correlation between DWI-ASPECTS and the immune status of patients with AIS.
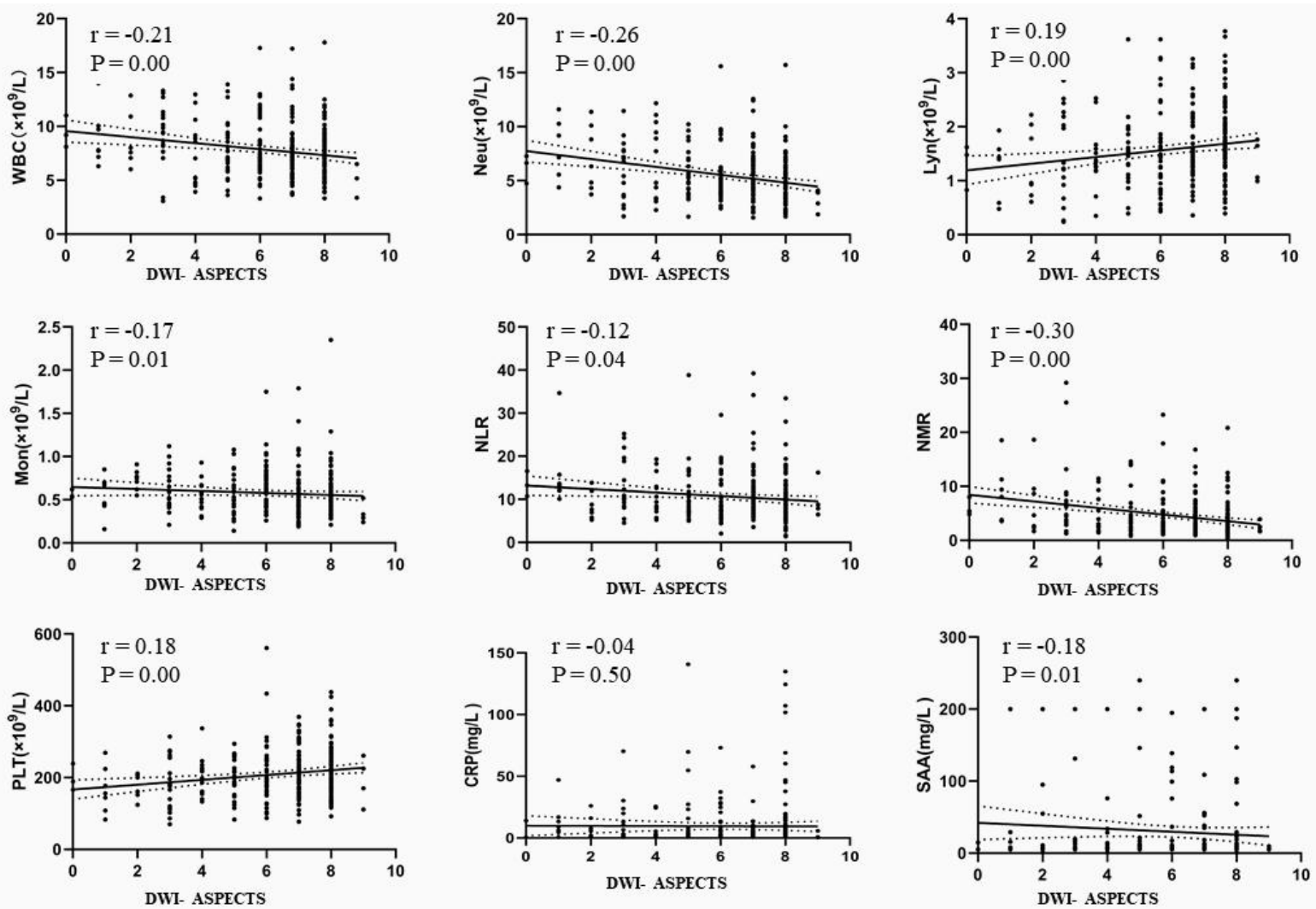